\title{
A biologically inspired control approach for distributed elastic systems
}

\author{
C. R. Fuller and J. P. Carneal \\ Vibration and Acoustics Laboratories, Mechanical Engineering Department, Virginia Polytechnic Institute \\ and State University, Blacksburg, Virginia 24061-0238
}

(Received 18 May 1992; revised 4 November 1992; accepted 20 January 1993)

\begin{abstract}
A multi-degree of freedom control approach, which is largely inspired by biological systems, is presented. Control inputs to a structure are achieved by multiple piezoelectric actuators. One actuator chosen as the "master" actuator is under the direction of the central, sophisticated controller. The other "slave" actuators derive their control inputs by localized, simple learning rules related to the behavior of their neighbor actuators including the "master." Simulations on the control of the vibrational energy density of a harmonically excited simply supported beam demonstrate a significant improvement in control performance over a single actuator case, particularly for off-resonance frequencies. Thus high attenuations are achieved with a multiple degree of freedom actuator with a single main channel of control.
\end{abstract}

PACS numbers: $43.40 . \mathrm{Vn}, 43.40 . \mathrm{At}$

\section{INTRODUCTION}

Recent work has demonstrated the potential of active control of distributed elastic systems using multiple, independent actuators and sensors. In work concerned with the control of sound radiation from vibrating panels, the importance of number of channels of control and optimization of the transducer position and shape has been demonstrated. ${ }^{1}$ However these works were carried out for a fixed frequency and it is apparent that for good control over a bandwidth of frequencies, the control actuators and sensors need to be adaptive in shape. At first sight this problem could be solved using an overall transducer broken up into many individual small elements each connected by an individual control channel. In this situation the control transducer will effectively re-optimize its configuration for different conditions by adaptively weighting each transducer segment. Meirovitch and Norris ${ }^{2}$ have demonstrated the advantage of such an approach by considering fully distributed control in reducing control spillover. The disadvantage of this approach is that, for systems with a high modal density, the number of actuators and sensors required becomes extremely large. This high number of control channels has a number of problems mainly associated with memory requirements and computational time in the hardware systems used to implement the control.

In this Letter we discuss a new approach to the control of the vibrational response of distributed elastic systems using a high number of channels of control. The approach is inspired by biological muscle control where a low number of main signals are transmitted from the brain to a large area of muscle tissue. The signals then stimulate local action that is governed by, for example, chemical interaction of locally connected nerves, etc., resulting in multiple subsequent signals for individual muscle cell elongation or contraction. Put simply, a signal is sent from a central complex processor (the brain) and then is broken into multiple signals by local simplified control rules (muscle cells, etc.). ${ }^{3}$

In the present study, we attempt to implement such a process. The system to be controlled is a simply supported beam harmonically excited by a point force and controlled by multiple piezoelectric elements bonded to the beam as shown in Fig. 1. One actuator is chosen as the "master" actuator (based on some knowledge of the system response) and is under direction of the main, centralized advanced controller. The other "slave" actuators derive their control inputs by localized, simple learning rules related to the behavior of their neighbor actuators and are independent of the main controller direct signal. For lack of a better name, we term this a "biologically inspired" control approach. Note that this approach is significantly different than those using genetic control algorithms such as investigated by Curtis. ${ }^{4}$

\section{ANALYSIS}

For the following analysis the input disturbance is harmonic and narrow band. The out-of-plane displacement response to the disturbance can be written as

$$
w_{f}(x, t)=-\frac{2 F_{d}}{M} \sum_{n=1}^{\infty} \frac{\sin \left(n \pi x_{d} / L\right) \sin (n \pi x / L) e^{-i \omega t}}{\left(\omega^{2}-\omega_{n}^{2}\right)},
$$

where $\omega_{n}$ is the natural frequency of the $n$th mode.

The out-of-plane response to a piezoelectric control actuator can be written as ${ }^{5}$

$$
\begin{aligned}
w_{p}(x, t)= & -V \sum_{n=1}^{\infty} C_{n} \frac{\cos \left(n \pi x_{2} / L\right)-\cos \left(n \pi x_{1} / L\right)}{\left(\omega^{2}-\omega_{n}^{2}\right)} \\
& \times \sin \left(\frac{n \pi x}{L}\right) e^{-i \omega t},
\end{aligned}
$$




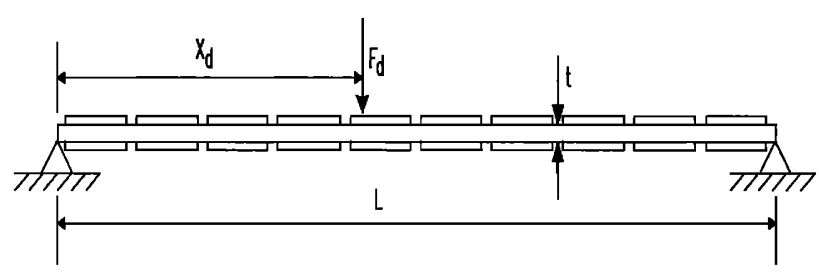

FIG. 1. Simply supported beam with multiple PZT actuators.

where $V$ is the applied voltage, $x_{1}$ and $x_{2}$ are the locations of the edge of the piezoelectric patch, and $C_{n}$ is a piezoelectric coupling constant given in Ref. 5 .

By superposition the total response of the beam can be written as

$$
w_{t}(x, t)=\left(w_{f}(x)+\sum_{j=1}^{J} w_{p}^{j}(x)\right) e^{-i \omega t},
$$

where $J$ is the total number of piezoelectric actuators employed.

We define a control cost function as the out-of-plane vibrational energy density function,

$$
\Lambda(V)=\int_{0}^{L}\left|\dot{w}_{t}\right|^{2} d x
$$

The objective is to implement a multi-channel control approach to minimize $\Lambda$. This is accomplished by splitting the control implementation into two steps, as shown in Fig. 2. First, we optimize with the main controller and then local learning rules are used.

\section{A. Main controller}

Initially the main controller minimizes the vibrational energy density by using linear quadratic optimal control theory to derive the optimal voltage input to the "master" actuator. In practice, this would be achieved with an adaptive LMS control approach. The optimal voltage is given, for a SISO system, ${ }^{1}$ by

$$
V_{\mathrm{opt}}=-\frac{H_{d e}(\omega)}{H_{p e}(\omega)} F_{d},
$$

where $H_{d e}(w)$ is the influence function between the disturbance force and the error signal, defined as

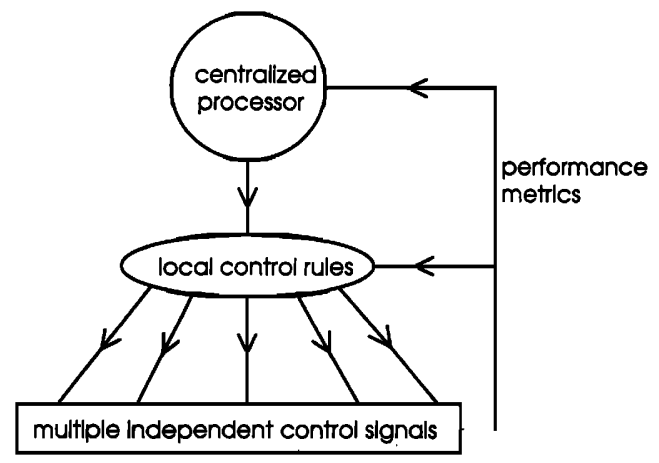

FIG. 2. Biological control approach.
TABLE I. Beam natural frequencies $(\mathrm{Hz})$.

\begin{tabular}{cc}
\hline \hline Mode & Frequency $(\mathrm{Hz})$ \\
\hline 1 & 46.5 \\
2 & 186.1 \\
3 & 418.8 \\
\hline
\end{tabular}

$$
H_{d e}(\omega)=\frac{2}{M} \sum_{n=1}^{\infty} \frac{\sin \left(n \pi x_{d} / L\right)}{\left(\omega^{2}-\omega_{n}^{2}\right)}
$$

and $H_{p e}(w)$ is the influence function between the piezoelectric actuator voltage and the error signal, defined as

$$
H_{p e}(\omega)=\sum_{n=1}^{\infty} C_{n} \frac{\cos \left(n \pi x_{2} / L\right)-\cos \left(n \pi x_{1} / L\right)}{\left(\omega^{2}-\omega_{n}^{2}\right)} .
$$

\section{B. Local control approach}

Once we have found the optimal voltage to the "master" actuator, the control voltages to the "slave" actuators are derived using local learning rules. The following example approach for a local learning rule is based on the observation that, for a distributed elastic system with little damping, regions are close to either in or out-of-phase with a fixed point. Thus the local learning approach is to take the "master" control voltage and apply it to the neighbor slave actuator (i.e., actuator immediately alongside). The control voltage is tried in-phase $(+1)$, out-of-phase $(-1)$, or turned off $(0)$ while the cost function $\Lambda$ is observed for each change. The condition that causes the cost function to be reduced the most is kept and the process is then applied to the next neighbor "slave" actuator until all elements are progressively tested. By this method, a distributed actuator with a generalized function that drives a response similar to the uncontrolled vibration distribution with low control spillover is constructed. Note that the previous process is sub-optimal and alternative local learning strategies could be developed.

\section{EXAMPLE RESULTS}

For an illustrative example, we consider a steel beam of length $L=0.5 \mathrm{~m}$, thickness $t=5 \mathrm{~mm}$ driven by an input point force disturbance $F_{d}$ of unity magnitude $(N)$ at position $x_{d}=0.200 \mathrm{~m}$. Natural frequencies of the beam are given in Table I. Ten piezoelectric actuators are used with element lengths of $49.1 \mathrm{~mm}$ and spacing gaps of $1 \mathrm{~mm}$. The seventh actuator from the left is chosen as the single "master" actuator. For the first case the input frequency is $\mathbf{5 5 2}$ $\mathrm{Hz}$, which is off resonance between the third and fourth mode resonance points of the beam. Figure 3 shows the beam rms, response for no control, the "master" actuator alone and control using the "master" actuator and localized learning rules to derive "slave" actuator control voltages. Table II also presents the corresponding attenuation of the cost function for both cases as well as the string of control inputs to the slave actuators derived by the localized rules.

As can be seen, the single "master" actuator provides only $1.0 \mathrm{~dB}$ of attenuation and leads to significant spillover 


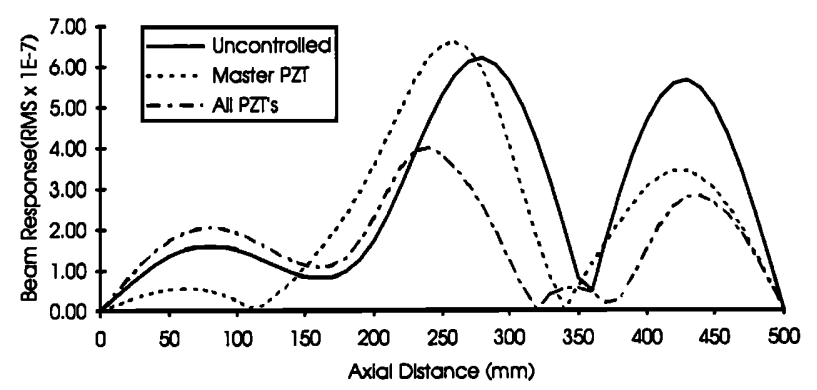

FIG. 3. Off-resonance beam excitation $(552 \mathrm{~Hz})$.

in the region of the beam 150 to $275 \mathrm{~mm}$. With the use of the multiple "master" and "slave" actuators the attenuation is improved to $4.6 \mathrm{~dB}$. Improved control in the region of largest response is now achieved with little control spillover. The string of control inputs show that many of the control actuators are not implemented at all, while control actuation is centered around the region of largest response.

The input frequency was now lowered to $80 \mathrm{~Hz}$, which is off resonance between the first and second mode resonance points. Figure 4 shows the beam displacement with and without control and again improved performance is demonstrated using the multiple piezoelectric actuators optimized using local learning rules. Table II also predicts an increase of around $5 \mathrm{~dB}$ in attenuation of the cost function when all actuators are used. In this case, it is apparent that more "slave" actuators are required to constrain the control spillover. Note that the optimal inputs are symmetric corresponding to the nearly symmetric uncontrolled response.

For the last case considered the frequency was set to $183 \mathrm{~Hz}$, which is close to the second mode resonance point. The displacement results demonstrate high control of the beam response with little difference between using the single "master" actuator and all the actuators together. Table

TABLE II. Control performance.

\begin{tabular}{cccc}
\hline \hline Freq. $(\mathrm{Hz})$ & Control string & "Master" (dB) & $\begin{array}{c}\text { "Master" and } \\
\text { "slaves" (dB) }\end{array}$ \\
\hline 552 & $0000-0 \oplus+00^{\mathrm{a}}$ & 1.0 & 4.6 \\
80 & $-00+00 \oplus 00-$ & 4.8 & 9.6 \\
183 & $000000 \oplus 000$ & 27.7 & 27.7 \\
\hline
\end{tabular}

aNote: $\oplus$ corresponds to "master" actuator.

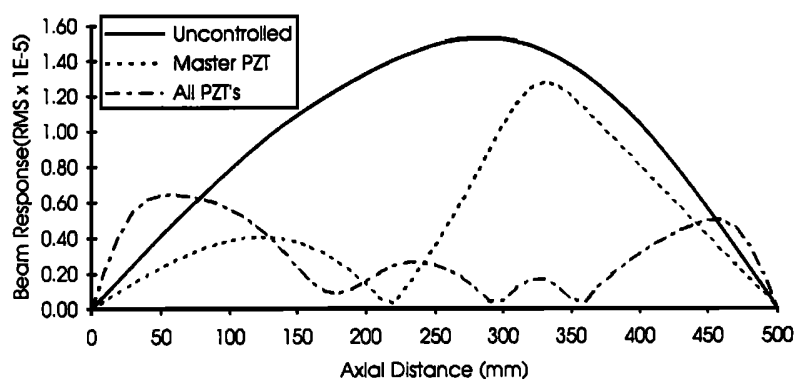

FIG. 4. Off-resonance beam excitation $(80 \mathrm{~Hz})$.

II shows that, in fact, all the slave actuators are set to zero using the localized learning rule approach due to the suboptimal nature of the procedure.

\section{CONCLUSIONS}

The potential of using a hybrid controller based on biological systems has been demonstrated. The key to the approach is to take advantage of known system behavior to use localized simple training rules in conjunction with a centralized, sophisticated controller. It has been demonstrated that a distributed actuator with many degrees of freedom under the direction of the centralized controller with a single control channel can be successfully implemented. The results of this limited study indicate the approach gives the highest performance benefits for offresonance excitation when the response modal density is high. Future work will consider 2-D systems and more general local training rules.

\section{ACKNOWLEDGMENTS}

The authors gratefully acknowledge the support of this work by NASA Langley Research Center and the Office of Naval Research.

${ }^{1}$ R. L. Clark, "Advanced Sensing Techniques for Active Structural Acoustic Control," Ph.D. dissertation, Virginia Polytechnic Institute and State University (1992).

${ }^{2}$ L. Meirovitch and M. A. Norris, "Vibration Control," in Proceedings of Inter-Noise 84 (Noise Control Foundation, Poughkeepsie, NY, 1984), pp. $477-482$.

${ }^{3}$ B. R. Landau, Essential Human Anatomy and Physiology (Scott, Foresman, Glenview, IL, 1976), pp. 191-192.

${ }^{4}$ A. R. D. Curtis, "An Application of Genetic Algorithms to Active Vibration Control," in Proceedings of Recent Advances in Active Control of Sound and Vibration (Technomic, Lancaster, PA, 1991), p. 338.

${ }^{5}$ R. L. Clark, C. R. Fuller, and A. L. Wicks, "Characterization of multiple piezoelectric actuators for structural excitation," J. Acoust. Soc. Am. 90, 346-357 (1991). 